BULLETIN OF THE

AMERICAN MATHEMATICAL SOCIETY

Volume 80, Number 5, September 1974

\title{
LEBESGUE SPACES FOR BILINEAR VECTOR INTEGRATION THEORY
}

\author{
BY JAMES K. BROOKS ${ }^{1}$ AND NICOLAE DINCULEANU ${ }^{2}$
}

Communicated by Robert Bartle, December 12, 1973

In this note we shall announce results concerning the structure of $L_{E}^{1}(m)$, the space of $E$-valued functions integrable with respect to a measure $m: \Sigma \rightarrow L(E, F)$, where $L(E, F)$ is the class of bounded operators from the Banach space $E$ into the Banach space $F$. The bilinear integration theory introduced here is more restrictive than the one developed by Bartle [1], but it is general enough to allow a norm to be defined on the integrable functions and to permit the study of weak compactness and convergence theorems; moreover, $L_{E}^{1}(m)$ lends itself in a natural way to the study of continuous operators $T: C_{E}(S) \rightarrow F$, where the domain is the space of continuous $E$-valued functions defined on the compact Hausdorff space $S$ as follows: By Dinculeanu's representation theorem [6], there exists a unique regular finitely-additive measure $m: \Sigma \rightarrow L\left(E, F^{* *}\right)$, where $\Sigma$ is the family of Borel subsets of $S$, such that $T(f)=\int f d m$. If $T$ is a weakly compact operator, Brooks and Lewis [2] have shown that $m$ is countably additive, with range in $L(E, F)$. In addition, the set $N=\left\{\left|m_{z}\right|: z \in F_{1}^{*}\right\}$ is relatively weakly compact in ca $(\Sigma)$-here $m_{z}$ is the $E^{*}$-valued measure defined by $m_{z}(A) e=\langle m(A) e, z\rangle$, and $\left|m_{z}\right|$ is the total variation function of $m_{z}$. Conversely, if $N$ has the above property and $E$ is reflexive, then $T$ is weakly compact. A natural question is whether a Lebesgue space $L_{E}^{1}(m) \supset C_{E}(S)$ of $m$-integrable functions can be defined. If so, what convergence theorems can be proved, and how are the weakly compact sets characterized?

The setting is as follows. Let $\Sigma$ be a $\sigma$-algebra of subsets of a set $T$, and $m: \Sigma \rightarrow L(E, F)$, a countably additive measure be given such that $m$ is strongly bounded, that is, $\tilde{m}_{E, F}\left(A_{i}\right) \rightarrow 0$, whenever $\left(A_{i}\right)$ is a disjoint sequence of sets $\left(\tilde{m}_{E, F}\right.$ is the semivariation of $m$ with respect to $E$ and $F$ [6]). It follows that $N=\left\{\left|m_{z}\right|: z \in F_{1}^{*}\right\}$ is relatively weakly compact in $\mathrm{ca}(\Sigma)$. Let $\lambda$ be a positive control measure for $m$ such that $\lambda \leqq \tilde{m}_{E, F}$ and

AMS (MOS) subject classifications (1970). Primary 46E30, $28 \mathrm{~A} 45$.

${ }^{1}$ Supported in part by NSF Grant GP 28617.

${ }^{2}$ Supported in part by NSF Grant GP $31821 X$.

Copyright @ American Mathematical Society 1974 
$m \ll \lambda$. The set $F_{E}(N)$ is the set of $\lambda$-measurable $E$-valued functions $f$ such that

$$
N(f)=\sup \left\{\int|f| d\left|m_{z}\right|: z \in F_{1}^{*}\right\}<\infty .
$$

For every $f \in F_{E}(N)$, define the integral $\int f d m \in F^{* *}$ by $\left\langle z, \int f d m\right\rangle=$ $\int f d m_{z}$, for $z \in F^{*}$. The closure of the $E$-valued simple functions in $F_{E}(N)$ is defined to be $L_{E}^{1}(m)$. By $L^{1}(m)$ we denote the Banach space of scalar $m$-integrable functions. The Vitali convergence theorem and the Lebesgue dominated convergence theorem are valid in $L_{E}^{1}(m)$, but the Beppo Levi theorem fails in general. We say that $m$ has the Beppo Levi property if every increasing sequence of positive simple functions $f_{n}$, with $\sup _{n} N\left(f_{n}\right)<\infty$, is a Cauchy sequence in $L^{1}(m)$. In Theorem 1 below, it is seen that this crucial property is satisfied under mild conditions. In the case $E$ is the scalar field, $L^{1}(m)$ includes the Bartle-DunfordSchwartz integral as defined in [7, Chapter IV].

The authors have developed a more general theory in which Lebesgue spaces $L_{E}^{1}(N)$ are studied, where $N$ is a family of positive measures not necessarily arising from a vector measure as above. The theory of associate spaces is introduced and representations of operators $T: L_{E}^{1}(N) \rightarrow F$ are given. To cover the case when $S$ is a locally compact space, we construct the theory on $\delta$-rings by means of localizable control measures. In this announcement we shall restrict ourselves to the above special case and a few representative theorems will be stated. The complete version of these results will appear in [4].

THEOREM 1. If $\int f d m \in F$ for every $f \in F_{E}(N)$, then $m$ has the Beppo Levi property. In particular, if $F$ is weakly sequentially complete, then $m$ has the Beppo Levi property.

INDICATION OF THE PROOF. One can show that it is sufficient to prove that $m$ has the Beppo Levi property if whenever $f \in F_{E}(N)$ and $A_{n} \searrow \varnothing$, then $N\left(f \chi_{A_{n}}\right) \rightarrow 0$. If we deny this, there exist $z_{n} \in F_{1}^{*}$ such that $\int_{A_{n}}|f| d\left|m_{z_{n}}\right|>\varepsilon>0$ for every $n$, for some $\varepsilon$. By proving that

$$
\int_{A_{n}}|f| d\left|m_{z_{n}}\right|=\sup \left\{\left|\int_{A_{n}} g d m_{z_{n}}\right|, g \text { is simple, }|g| \leqq|f|\right\},
$$

we may assume: $(*)\left|\int_{A_{n}} f_{n} d m_{z_{n}}\right|>\varepsilon, n=1,2, \cdots$, where the $f_{n}$ are simple $E$-valued functions. Form $L_{E_{0}}^{1}\left(\Sigma_{0}, m\right)$, where $\Sigma_{0}$ is a separable $\sigma$-algebra containing $\left(A_{n}\right)$ such that $f$ and $f_{n}$ are $\Sigma_{0}$-measurable; $E_{0}$ is the closure of the span of $f(T) \cup \bigcup_{n \geqq 1} f_{n}(T)$. Let $F_{0} \subset F$ be the separable Banach space generated by $\left\{\int h d m: h \in L_{E_{0}}^{1}\left(\Sigma_{0}, m\right)\right\}$. By using the Lebesgue dominated convergence theorem in the spaces $L_{E_{0}}^{1}\left(\left|m_{z}\right|\right)$, we see that $h \in F_{E_{0}}(N)$ 
implies $\int h d m \in F_{0}$, whenever $h$ is $\Sigma_{0}$-measurable. By a diagonal process, assume that $\left(z_{n}\right)$ converges on a countable dense subset of $F_{0}$; hence $\left(z_{n}\right)$ converges on $F_{0}$. Let $\tau=\Sigma 2^{-n}\left|m_{z_{n}}\right|$, and define $A\left(\Sigma_{0}, f\right)$ to be the space of $\Sigma_{0}$-measurable functions $h: T \rightarrow E_{0}$ such that $|h| \leqq|f|$ a.e. $\tau$; thus $A\left(\Sigma_{0}, f\right)$ is a complete subset of $L_{E_{0}}^{1}\left(\Sigma_{0}, \tau\right)$. Define $T_{n}: L_{E_{0}}^{1}\left(\Sigma_{0}, \tau\right) \rightarrow$ $F_{0}$ by $T_{n}(h)=\int h d m_{z_{n}}$; note that $\left\|T_{n}\right\| \leqq 2^{n}$. It follows that $\lim T_{n}(h)$ exists for every $h \in A\left(\Sigma_{0}, f\right)$. Using the Baire category theorem, we deduce that $\left(T_{n}\right)$ is equicontinuous at zero on $A\left(\Sigma_{0}, f\right)$. However, since $\int_{A_{n}}\left|f_{n}\right| d \tau \leqq \int_{A_{n}}|f| d \tau \rightarrow 0$, we have $\lim _{n} \int_{A_{n}} f_{n} d m_{z_{k}}=0$ uniformly in $k$. This contradicts $(*)$.

Using the above theorem, we are able to give sufficient conditions for a set to be relatively weakly compact in $L_{E}^{1}(m)$.

THEOREM 2. Assume that $E$ is reflexive and $F$ is weakly sequentially complete. Suppose $K \subset L_{E}^{1}(m)$ is a set satisfying:

(1) $K$ is bounded;

(2) $N\left(f \chi_{A_{n}}\right) \rightarrow 0$ uniformly for $f \in K$, whenever $A_{n} \searrow \varnothing$.

Then $K$ is relatively weakly compact.

INDICATION OF THE PROOF. By the Eberlain-Smulian theorem, we may assume that $K$ is a sequence of functions $f_{n}$. Let $\lambda$ be a bounded control measure for $m$; hence $\lambda(A) \leqq N\left(\chi_{A}\right)$, for $A \in \Sigma$, and $N \ll \lambda$ uniformly. We assert that $\lim _{N(A) \rightarrow 0} N\left(f \chi_{A}\right)=0$ uniformly for $f \in K$. By hypothesis the set $Q=\left\{\int|f| d\left|m_{z}\right|: z \in F_{1}^{*}\right\}$ is uniformly countably additive. Also each measure in $Q$ is absolutely continuous with respect to $\lambda$. By [3, Theorem 2.1] $Q \ll \lambda$ uniformly. This implies the above assertion. Since $\lambda \leqq N$, we see that $K$ is a bounded subset of $L_{E}^{1}(\lambda)$; moreover, $\lim _{\lambda(A) \rightarrow 0} \int_{A}|f| d \lambda=0$ uniformly for $f \in K$. Hence $K$ is relatively weakly compact in $L_{E}^{1}(\lambda)$ [5], [3]. Assume that $\left(f_{n}\right)$ converges weakly to $f_{0} \in L_{E}^{1}(\lambda)$; we shall show that $\left(f_{n}\right)$ converges weakly to $f_{0}$ in $L_{E}^{1}(m)$. It can be shown that $L_{E}^{1}(m)^{*}$ consists of $E^{*}$-valued measures of finite total variation, absolutely continuous with respect to $\lambda$. Let $\sigma \in L_{E}^{1}(m)^{*}$. Using the Phillips theorem, we prove that there exists a $g \in L_{E^{*}}^{1}(m)$ such that $\sigma(h)=\int g h d \lambda$, for $h \in L_{E}^{1}(m)$. The sequence $\left(g f_{n}\right)$ is bounded in $L^{1}(\lambda)$, since $\left\|g f_{n}\right\|_{1} \leqq\|\sigma\| N\left(f_{n}\right)$; note also that $\lim _{\lambda(A) \rightarrow 0} \int_{A}\left|g f_{n}\right| d \lambda=0$ uniformly in $n$. As a result, $\left(g f_{n}\right)$ is relatively weakly compact in $L^{1}(\lambda)$. Thus we may assume that $\left(g f_{n}\right)$ converges weakly to $h \in L^{1}(\lambda)$. Let $\left(B_{k}\right)$ be a sequence of sets with union equal to $T$ such that $g$ is bounded on each $B_{n}$. For fixed $k, g \chi_{C} \in L_{E^{*}}^{\infty}(\lambda)=L_{E}^{1}(\lambda)^{*}$, for every $C \in \Sigma \cap B_{K^{*}}$. Thus $\int_{C} g f_{n} d \lambda \rightarrow \int_{C} g f_{0} d \lambda$ and $\int_{C} f_{n} g d \lambda \rightarrow \int_{C} h d \lambda$. Consequently $g f_{0}=h$ a.e. $\lambda$ on $B_{k}$, hence $g f_{0}=h$ a.e. $\lambda$ on $T$. Therefore: $(\#) g f_{0} \in L^{1}(\lambda)$ for every $g \in L_{E}^{1}(m)^{*}$. To show that $f_{0} \in L_{E}^{1}(m)$ we proceed in two stages. First of all, we define the "associate space" $F_{E}{ }^{*}\left(N^{\prime}\right)$ of $F_{E}(N)$ to be the space of $\lambda$-measurable 
functions $g: T \rightarrow E^{*}$ such that $g f \in L^{1}(\lambda)$ for every $h \in L_{E}^{1}(m)$. Then we show that when $m$ has the Beppo Levi property (which, by Theorem 1 , it does in this instance), then $f_{0} \in L_{E}^{1}(m)$ if and only if $g f_{0} \in L^{1}(\lambda)$ for every $g \in F_{E^{*}}\left(N^{\prime}\right)$. Then we prove that $F_{E^{*}}\left(N^{\prime}\right)=L_{E}^{1}(m)^{*}$. The lengthy details are omitted. This, in conjunction with (\#), implies that $f_{0} \in L_{E}^{1}(m)$.

COROLLARY 1. Let $F$ be weakly sequentially complete. Suppose $\left(f_{n}\right)_{n \geqq 0} \subset$ $L^{1}(m)$. If $\int_{A} f_{n} d m \rightarrow \int_{A} f_{0} d m$ for every $A \in \Sigma$, then $f_{n} \rightarrow f_{0}$ weakly in $L^{1}(m)$.

From the construction in the proof of Theorem 2, we deduce the following corollary.

COROLlaRY 2. If $E$ is reflexive and $F$ is weakly sequentially complete, then $L_{E}^{1}(m)$ is weakly sequentially complete.

\section{REFERENCES}

1. R. G. Bartle, A general bilinear vector integral, Studia Math. 15 (1956), 337-352. MR 18, 289.

2. J. K. Brooks and P. W. Lewis, Operators on function spaces, Bull. Amer. Math. Soc. 78 (1972), 697-701. MR 45 \#7494.

3. J. K. Brooks and N. Dinculeanu, Strong additivity, absolute continuity and compactness in spaces of measures, J. Math. Anal. Appl. 45 (1974), 156-175.

4. - Lebesgue-type spaces for vector integration. Linear operators, weak completeness and weak compactness, J. Math. Anal. Appl. (to appear).

5. S. D. Chatterji, Weak convergence in certain special Banach spaces, MRC Technical Sum. Report \#443, University of Wisconsin, Madison, Wis., 1963.

6. N. Dinculeanu, Vector measures, Internat. Ser. of Monographs in Pure and Appl. Math., vol. 95, Pergamon Press, Oxford, 1967. MR 34 \#6011b.

7. N. Dunford and J. T. Schwartz, Linear operators. I: General theory, Pure and Appl. Math., vol. 7, Interscience, New York, 1958. MR 22 \#8302.

Department of Mathematics, University of Florida, Gainesville, Florida 32601

Department of Mathematics, University of Bucharest, Bucharest, Romania 